\title{
A Distributed, Net Oriented Semantics for Delta Prolog
}

\author{
Antonio Brogi \& Roberto Gorrieri \\ Dipartimento di Informatica - Universita di Pisa \\ Corso Italia,40 - 1-56100 Pisa, Italy
}

\begin{abstract}
A truly distributed operational semantics for Concurrent Logic Languages is defined here, differently from those semantics based on intrinsically sequential, interleaving models defined so far. Delta Prolog and the underlying Distributed Logic are taken as case studies, in order to semantically denote AND-parallelism and cooperation via explicit communication mechanism (local environment model of computation). A scheme for translating a Delta Prolog system into a 1-safe Petri net is given and properties of (perpetual) processes based on the notion of causality, e.g. fairness and deadlock, are addressed.
\end{abstract}

\section{INTRODUCTION}

The Horn Clause Language (HCL for short) is a subset of the First Order Logic which has been extensively studied and has generated the family of Logic Languages. The computation of a HCL program consists in proving a goal $\mathrm{G}_{1}, \ldots, \mathrm{G}_{\mathrm{n}}$ by applying the clauses of the program. At each step of derivation there are two choices to be taken:

i) which goal $\mathrm{G}_{\mathrm{i}}$ to reduce (computation rule)

ii) which clause $C_{j}$ to apply to the selected goal (search rule)

This fact leads to implicit forms of potential parallelism. AND-parallelism exploits the parallelism in the choice of the goal to be reduced, i.e. all the goals can be derived in parallel (parallel computation rule). OR-parallelism is the parallelism in the clause selection phase, i.e. all the clauses whose head is unifiable with the goal are applied in parallel (parallel search rule). While models exploiting pure OR-parallelism do not present many problems because of the complete independence of the environments of the various branches of the OR-derivation, pure AND-parallelism presents several difficulties mainly due to the presence of shared logical variables.

As hardware VLSI technologies evolve, new highly parallel computer architectures become realizable which call for new languages for parallel programming. Logic languages are regarded as candidates for this purpose for their clean semantics and their implicit parallelism. Nevertheless, a parallel language requires some explicit mechanism for the control of concurrency: pure logic languages lack of synchronization mechanisms. This fact has led to two different approaches. The first one restricts the possible modes of the mechanism of unification by introducing an explicit distinction between producers and consumers of the values of a logical variable. The resulting model is a subcase of AND-parallelism, called STREAMparallelism [7] based on an eager evaluation of structured data which can be treated as streams. Many concurrent logic languages based on STREAM-parallelism have been proposed, among which we mention 
Concurrent Prolog [26], PARLOG [6] and Guarded Horn Clauses [27]. These languages introduce different types of constraints on the unification mechanism: Concurrent Prolog has read only annotations on variables, PARLOG provides mode declarations, while GHC solves the problem with the input guards.

The other approach extends HCL by introducing explicit operators like fork and join, proposed in [18,9,12], and communication primitives for message-passing (send/receive), as in Delta Prolog [1,20,21,22].

We study the case of pure AND-parallelism where the cooperation between parallel goals is performed via explicit mechanisms of communication / synchronization. While this model of computation represents the correspondent of the message-passing model in the field of traditional concurrent languages (and therefore is naturally suitable for distributed descriptions), STREAM-parallelism corresponds to a shared memory model of computation. In Delta Prolog, the cooperation mechanism is based on the notion of event, originally introduced in [5,17]. These features of Delta Prolog make it a good candidate as a distributed logic language.

The presence of extra-logical primitives for the explicit control of concurrency decreases the logical flavour of concurrent logic languages. Phenomena such as synchronization, communication, deadlock and process creation may be more advantageously modeled using techniques stemming from imperative and functional concurrent languages, emphasizing control rather than logic. In fact, interleaving based operational semantics have been recently defined: in [25], Saraswat has provided operational semantics for several concurrent logic languages by means of transition systems defined in the Structural Operational Semantics style [23] (SOS for short). Also [14] has addressed this problem in a similar way for a subset of Theoretical Flat Concurrent Prolog by using techniques developed for TCSP in [5]. In a recent paper ${ }^{[3]}$ an operational semantics has been given to Theoretical Concurrent Prolog resorting to techniques based on metric spaces.

A distributed, net oriented semantics for a concurrent logic language is presented here, to the best of our knowledge, for the first time. We model a concurrent system as a set of sequential processes, possibly located in different places, which cooperate in accomplishing a task: thus, neither a global state nor a global clock must be assumed. Behaviours of systems are represented through the causal relations among the events performed by subparts of their distributed states and then translated to a class of Petri nets [4,24], called 1-safe P/T nets. Petri nets are essentially nondeterministic automata enriched by giving states an additional structure of set in order to represent distributed states and by allowing transitions involving only some of the processes present in the actual state. The semantical description corresponds to an implementation where the goal is distributed on many processors, each containing a copy of the program.

In this paper we use a three step method. First, we describe the behaviour of sequential processes by means of two transition systems: one for programs and the other for sequential goals. The former describes the mechanisms of head unification and clause selection. The latter represents the evolution of sequential goals, possibly resorting to the program transition system when an atom goal needs a clause to unify with. Secondly, we represent the states of the concurrent system by sets of sequential processes and the evolution of the whole system is described in terms of process interactions. Finally, we map all our semantical structure in Petri Nets in quite a trivial manner. This constructive technique has been inspired by paper [11], where it was originally proposed, and $[8,10,19]$, where it has been applied to concurrent languages.

As a matter of fact, we stipulate a sort of well-formedness of goals with respect to communications. More precisely, we define a condition of closure, which states that a goal must be "communication closed" at the top-level, i.e. unable to perform an event-communication with an external partner. Since we are considering programs and goals ready to be executed, we exclude possible open communications which have a sense 
only during the composition of subsystems.

The classical operational semantics for logic languages denotes the set of solutions of a program as the set of computed answer substitutions. In our operational semantics, this success set together with the finite failures and the global deadlocks that may occur in a computation are observable from its final state. We could be dissatisfied for this final state semantics since it is not adequate w.r.t. infinite computations (perpetual processes) in the sense that relevant features of behaviours are not dealt with. A better description of a concurrent system should take into account all the actions performed (thus we know what the system is doing) and their causal relations, i.e. every computation is observed as the partial ordering of events it generates. This way, important properties, such as safety and liveness, can be observed.

Our semantical definitions do respect Delta Prolog specifications [1], nevertheless we do not compare our semantics with those of the authors of Delta Prolog since their operational model is interleaving while our one is distributed. It is worth observing that, with respect to the problem of dealing with distributed backtracking, our operational semantics is as far from implementation issues as the classical HCL operational semantics [28].

An outline of the paper follows. Section 2 contains the abstract syntax of Delta Prolog. The transition systems for programs and sequential goals are described in Section 3. In Section 4 we introduce a decomposition function in order to translate a goal into the set of its sequential processes. Then, we give a rewriting system for describing the interactions among sequential processes, and finally we consider closed systems. Section 5 contains the translation of the semantical structure into a 1-safe P/T Petri net, while several comments about semantics and properties of perpetual processes are addressed in Section 6.

\section{PRELIMINARIES}

We define the abstract syntax for a version of Delta Prolog $[1,20,22]$. The operators on goals resemble those of other concurrent languages such as CCS [17], TCSP [5] and CSP [15]. In Delta Prolog (DP for short) a clear distinction is made between sequential ";" and parallel composition "II" at the syntactical level. In $\mathrm{HCL}$ the meaning of the operator "," can not be immediately turned to either one. It is not a sequential composition operator since the success set does not depend on the order, if any, atoms in a goal have to be selected (i.e. on the computation rule) nor it is a parallel operator since atoms can not be independently derived in parallel (because of shared variables). Moreover, an explicit construct for exchanging information is introduced. Subgoals of a parallel goal can communicate by event goals, labelled by an event name representing the name of a communication channel.

The language alphabet is $\langle D, V, P \cup C$, where $D$ is a family indexed on $\mathbf{N}$ (natural numbers) of data constructors $\left(D_{0}\right.$ is the set of constants), $V$ represents the set of variable symbols, $P$ the set of all program-defined predicate symbols and $C$ the set of (unary, postfixed) communication predicate symbols $\left\{? \mathrm{e}\right.$, !e $\left.\mid \mathrm{e} \in D_{0}\right\}$. In the following, let $\mathrm{A}$ represent a program-defined atomic formula and $\mathrm{T}$ a term in $T_{D(V) / \approx}$ (the free $D$-algebra on $V$ modulo variance).

Definition 2.1. (Delta Prolog Syntax)

The abstract syntax of Delta Prolog is given by the following BNF-like grammar:

$$
\begin{aligned}
& P::=A \leftarrow|A \leftarrow G| P \oplus P \\
& G::=A\left|G_{e}\right| G_{C}|G ; G| G \| G \\
& G_{e}::=T ? \mid T ! e \\
& G_{c}::=G_{e} ; G \mid G_{c} \therefore G_{c}
\end{aligned}
$$


We denote by:

$\operatorname{Pr}$ the set of programs, ranged over by $\mathrm{p}$ (possibly indexed)

$G$ the set of goals, ranged over by $g$ (possibly indexed)

$G_{e}$ the set of event goals, ranged over by $\mathrm{g}_{\mathrm{e}}$ (possibly indexed)

$G_{c}$ the set of choice goals, ranged over by $\mathrm{g}_{\mathrm{c}}$ (possibly indexed)

A goal $g$ is a parallel goal iff it has either form:

$$
\mathrm{g}=\mathrm{g}_{1} \| \lg _{2} \quad \mathrm{~g}=\mathrm{g}^{\prime} ; \mathrm{g}^{\prime \prime} \quad \text { where } \mathrm{g} \text { ' is a parallel goal. }
$$

Otherwise $\mathrm{g}$ is a sequential goal.

Let us give an informal overview of DP syntax and semantics. A program is a fact $(A \leftarrow)$, a definite clause $(A \leftarrow G)$ or the union of two programs $(P \oplus P)$ : in other words a program is a set of facts and clauses. $A$ goal $g$ can be an atom $\mathrm{A}$, an event goal $\mathrm{g}_{\mathrm{e}}$, a choice goal $\mathrm{g}_{\mathfrak{c}}$, the sequential composition or the parallel composition of two goals. There are two types of events goals with the form, respectively: T?e and T!e, where $T$ is a term (the message), e is the event name (the communication channel), "!" and "?" specify the communication mode (send/receive). In order to solve an event goal T?e, we have to solve simultaneously a complementary event goal $T$ 'le. Moreover, both goals can solve if and only if $T$ and $T$ ' unify and, after the unification is performed, they become ground [1]. As an example, the two event goals: 1le and 2?e do not match as well as $X$ !e and $Y$ ?e, while $p(1, y) ! e$ and $p(x, 2)$ ?e do match by generating an exchange of values, i.e. the substitution $\{x / 1, y / 2\}$. Summing up, every communication is due to the simultaneous execution of exactly two complementary event goals, thus only symmetric, synchronous communication is allowed.

When dealing with a choice goal, only one goal is chosen for the rest of the derivation. If both goals could generate a step of derivation, one of them is nondeterministically selected. The operator of sequentialization ";" simply states that in $\mathrm{g}_{1} ; \mathrm{g}_{2}, \mathrm{~g}_{1}$ has to be solved before $\mathrm{g}_{2}$.

Finally, in order to solve a parallel goal $\mathrm{g}_{1} \| \mathrm{g}_{2}$ we solve $\mathrm{g}_{1}$ and $\mathrm{g}_{2}$ in parallel. In contrast with the fact that Delta Prolog is based on a message-passing model of computation, it allows variable sharing by parallel goals. Two goals are independently solved in parallel generating local bindings. When both processes have terminated, the two environments are joined, i.e. we verify if the independently computed substitutions are compatible.

The target of our semantical definition is to model the peculiar aspects of control operators for concurrency. Therefore we do not deal with clause order or cut operator, i.e. we refer to HCL instead of Prolog as the support for Delta Prolog, as it is specified in the original proposal of Distributed Logic. It is worth observing that a complete definition of the transition system for Prolog with cut operator can be easily given [25].

\section{The Transition Systems for Programs and Sequential Goals}

We define the operational semantics in the Structural Operational Semantics style [23] as a hierarchy of abstract machines. The lower level machine, described as a transition system, defines the mechanisms of head unification and clause selection, i.e. the program dependent features. Then, an abstract machine for sequential goals is presented, again as a transition system possibly resorting to the former transition system when an atom goal needs a clause to unify with. Finally, a rewriting system representing the evolution of the whole system is introduced. A state is represented as a set of sequential processes, while the rewriting rules describe process interactions. 
Definition 3.1. (Labelled Transition System)

A labelled transition system is a triple $\langle\Gamma, \Lambda, \rightarrow\rangle$, where $\Gamma$ is a set of configurations, $\Lambda$ is the set of labels and $\rightarrow \subseteq \Gamma \times \Lambda \times \Gamma$ is the transition relation. A configuration $\gamma$ is called stuck iff $\forall \lambda \in \Lambda, \forall \gamma^{\prime} \in \Gamma:\left\langle\gamma, \lambda, \gamma^{\prime}\right\rangle \notin \rightarrow$.

We now define the sets of labels and configurations for programs and sequential goals. Since we give semantics to a pair sprogram, goal», we should include the program into every configuration. However, since the program does never change during the evaluation of the goal, we do not insert it in all the configurations, but just consider it as an implicit parameter.

Definition 3.2. (Labels and Configurations)

We define the sets of labels and configurations for sequential goals and programs as follows.

Let $\mathrm{g} \in G$ be a goal, $\mathrm{A} \in$ Atom, $\mathrm{p} \in \operatorname{Pr}, \theta \in S u b^{1}, \square$ the empty goal, $\mathrm{T}$ a term and failure a special symbol.

$$
\begin{array}{ll}
\Lambda_{\mathrm{G}}=\{\theta\} \cup\{\mathrm{T} ! \mathrm{e}\} \cup\{\mathrm{T} ? \mathrm{e}\} & \Lambda_{\mathrm{P}}=\{\theta\} \\
\Gamma_{\mathrm{G}}=\langle\mathrm{g}, \theta\rangle \cup\langle\mathrm{D}, \theta\rangle \cup \text { failure } & \Gamma_{\mathrm{P}}=\Gamma_{\mathrm{G}} \cup\langle\mathrm{A} ; \mathrm{p}, \theta\rangle
\end{array}
$$

These sets are ranged over by $\lambda_{G}, \lambda_{P}, \gamma_{G}$ and $\gamma_{P}$, respectively, omitting the subscripts whenever unambiguous.

A configuration $\langle\mathrm{g}, \theta\rangle$ describes a goal $\mathrm{g}$ to be reduced and a substitution $\theta$ representing the history of the whole derivation from the initial goal to g. Every transition is labelled with the substitution computed in the last step of derivation or by an event goal. In order to describe the communication mechanism, we use labels of the form T!e, T?e. When a sequential goal communicates, it assumes the existence of a partner for the communication. The existence of such a partner will be checked only at composition time and thus we have to store the name, the communication mode (! or ?) and the term $\mathrm{T}$ of a not yet matched event goal. $\square, \theta\rangle$ stands for the special stuck configuration "nothing to do", while $\langle A$ :p, $\theta\rangle$ represents an atom goal A to be solved via program $\mathrm{p}$. When goal $\mathrm{A}$ is unable to make further progress because there is no unifiable clause, the configuration (failure is reached. Thus we can directly observe the case of failure and distinguish it from deadlock.

\subsection{Transitions for Programs}

The (possibly infinite) set of transitions is generated by means of inference rules defined through syntax driven rules in the SOS style. The problems of head unification and clause selection are program dependent [25]. We describe how a clause of the program is selected to unify its head with the goal. Note that a clause invocation fails if head unification fails, the activated goal fails only if all the clause invocations fail.

\section{Definition 3.3.}

The program derivation relation over configurations, written as $\gamma_{\mathrm{P}}-\lambda_{\mathrm{P}} \rightarrow>_{\mathrm{p}} \gamma_{\mathrm{P}}$, is defined as the least relation satisfying the following inference rules.

\section{Head Unification)}

$$
\begin{aligned}
& \left.\theta=\operatorname{mgu}\left(A_{1}, A_{2}\right) \quad \text { implies }\left\langle A_{1}: A_{2} \leftarrow g, \delta\right\rangle-\theta\right\rangle_{p}\langle g \theta, \delta \theta\rangle \\
& \text { and } \left.\left.\left\langle A_{1}: A_{2} \leftarrow, \delta\right\rangle-\theta\right\rangle_{p} \backsim, \delta \theta\right\rangle
\end{aligned}
$$

1 Sub denotes the set of substitutions. 


$\begin{array}{lll}\neg \exists \operatorname{mgu}\left(\mathrm{A}_{1}, \mathrm{~A}_{2}\right) & \text { implies } & \left.\left\langle\mathrm{A}_{1}: \mathrm{A}_{2} \leftarrow \mathrm{g}, \delta\right\rangle-\varepsilon \rightarrow\right\rangle_{\mathrm{p}}\langle\text { failure }\rangle \\ & \text { and } & \left\langle\mathrm{A}_{1}: \mathrm{A}_{2} \leftarrow, \delta\right\rangle \rightarrow \mathrm{E} \rightarrow \mathrm{p}\langle\text { failure }\end{array}$

\section{Clause Selection)}

$$
\begin{aligned}
& \langle A: p, \delta\rangle-\theta-\rangle_{p}\langle g, \delta \theta\rangle \quad \text { implies } \quad\left\langle A:\left(p \oplus p^{\prime}\right), \delta\right\rangle-\theta \rightarrow_{p}\langle g, \delta \theta\rangle \\
& \text { and } \left.\left\langle A:\left(p^{\prime} \oplus \mathrm{p}\right), \delta>-\theta \rightarrow\right\rangle_{\mathrm{p}}<\mathrm{g}, \delta \theta\right\rangle \\
& \langle A: p, \delta\rangle-\varepsilon \rightarrow\rangle_{p} \text { (failure and }\left\langle A: p^{\prime}, \delta\right\rangle-\varepsilon>_{p} \text { (failure } \\
& \text { implies } \quad A:\left(p \oplus p^{\dagger}\right), \delta>-\varepsilon \rightarrow p \text { <failure } \\
& \text { and } \quad A:\left(p^{\prime} \oplus \mathrm{p}\right), \delta>-\varepsilon \rightarrow \mathrm{p} \text { <failure }
\end{aligned}
$$

\section{REMARKS}

Head Unification) The first rule simply states that if there is a mgu for an atom and the head of a clause then the atom can be replaced by the body of the clause, instantiated with the generated substitution. If the head of a clause does not unify with the atom to be proved, the derivation of the atoms leads to the special configuration failure.

Clause Selection) This rule states that the choice of a clause unifying with the goal to be proved cuts off all the other clauses. With respect to the case of failure, the goal fails if and only if it is not possible to find a clause of the program which is head-unifiable with it, i.e. all the possible clause selections lead to failure.

\subsection{Transitions for Sequential Goals}

In the following definitions we use:

$$
\gamma_{0} \rightarrow \gamma_{1} / \ldots / \gamma_{\mathbf{n}} \quad \text { implies } \quad \gamma_{0}^{\prime} \rightarrow \gamma_{1}^{\prime} / \ldots / \gamma_{\mathbf{n}}^{\prime}
$$

as an abbreviation for the $\mathrm{n}$ rules:

$$
\gamma_{0} \rightarrow \gamma_{i} \quad \text { implies } \quad \gamma_{0}^{\prime} \rightarrow \gamma_{i}^{i} \quad \text { for } i=1, \ldots, n \text {. }
$$

\section{Definition 3.4.}

The sequential goal derivation relation over configurations, written as $\gamma_{G}=\lambda_{G} \Rightarrow \gamma_{G}$, is defined as the least relation satisfying the following inference rules.

\section{Goal Derivation)}

$\langle A: p, \theta\rangle-\sigma-\rangle_{p}\langle g, \theta \sigma\rangle /\langle\square, \theta \sigma\rangle /\langle$ failure $\rangle$

$$
\text { implies }\langle A, \theta\rangle=\sigma \Rightarrow\langle g, \theta \sigma\rangle /\langle\square, \theta \sigma\rangle / \text { failure }\rangle
$$

\section{Sequential Composition)}

$\langle\mathrm{g}, \delta\rangle=\lambda_{\mathrm{G}} \Rightarrow\langle\square, \delta \theta, /\langle\mathrm{g}\rangle, \delta \theta\rangle /$ (failure $\rangle$

\section{Event Goal)}

$$
\text { implies } \left.\left\langle\mathrm{g} ; \mathrm{g}^{\prime \prime}, \delta\right\rangle=\lambda_{\mathrm{G}} \Rightarrow\left\langle\mathrm{g}^{\prime \prime} \theta, \delta \theta\right\rangle /\left\langle\mathrm{g}^{\prime} ;\left(\mathrm{g}^{\prime \prime} \theta\right), \delta \theta\right\rangle / \text { failure }\right\rangle
$$

$\langle\mathrm{T} ! \mathrm{e}, \delta\rangle=\mathrm{T} ! \mathrm{e} \Rightarrow\langle\square, \delta\rangle \quad$ and $\quad\langle\mathrm{T} ? \mathrm{e}, \delta\rangle=\mathrm{T} ? \mathrm{e} \Rightarrow\langle\mathrm{Q}, \delta\rangle$

\section{Choice Goal)}

$$
\begin{array}{cl}
\left\langle\mathrm{g}_{\mathrm{c}}, \delta\right\rangle=\lambda_{\mathrm{G}} \Rightarrow\langle\square, \delta \theta\rangle /\langle\mathrm{g}, \delta \theta\rangle & \text { implies }\left\langle\mathrm{g}_{\mathrm{c}} \therefore \mathrm{g}_{\mathrm{c}}, \delta\right\rangle=\lambda_{\mathrm{G}} \Rightarrow\langle\square, \delta \theta\rangle /\langle\mathrm{g}, \delta \theta\rangle \\
\text { and } & \left.\left\langle\mathrm{g}_{\mathrm{c}} \therefore \mathrm{g}_{\mathrm{c}}, \delta\right\rangle=\lambda_{\mathrm{G}} \Rightarrow\langle\square, \delta \theta\rangle / \mathrm{g}, \delta \theta\right\rangle
\end{array}
$$

\section{REMARKS}

Goal Derivation) This rule simply states that an atom A has to be solved by means of the transition 
system for programs, and therefore clause selection and head unification are part of the next step. Note that $p$ has to be a new, renamed copy of the program.

Sequential Composition) This rule states that the left component has to be solved first, and that the derived substitution has to be applied to the right component, too. On the other side, if the derivation leads to a failure, the whole goal fails.

Event Goal) According to [1], in order to solve an event goal $\mathrm{T}_{1}$ ?e, we must simultaneously solve a complementary event goal $T_{2}$ ?e. Both goals solve if and only if $T_{1}$ and $T_{2}$ unify and then, after the value exchange, both $\mathrm{T}_{1}$ and $\mathrm{T}_{2}$ are ground terms. Therefore, we have to store all the relevant information in the label so that, when dealing with the process interaction, this condition can be checked.

Choice Goal) If a goal $\mathrm{g}_{\mathrm{c}}$ can proceed somehow, the choice goal evolves by discarding the alternative and following the derivation of $\mathrm{g}_{\mathrm{c}}$.

Finally, note that all the configurations whose left component is a parallel goal are stuck in the transition system for sequential goals.

\section{The REWRITING DERIVATION RELATION FOR PARALlel GOALS}

We now decompose a parallel goal into those subgoals representing its sequential processes, in the style of $[11,10]$. For instance, from goal gllg' we will obtain two subgoals glid and idlg' (tag "lid" records that subterm $\mathrm{g}$ is in the left context of a parallel composition, symmetrically tag "idl"). More precisely, a sequential process is composed of a sequential goal (possibly the empty goal $\square$ or failure), a computed substitution, the access path codifying its location in the abstract syntax tree of a parallel goal (i.e. a structure of tags idl and lid), and a continuation specifying what to do afterwards. Now, the evolution of a set of sequential processes $I$, included in a state, is represented by a rewriting rule of the form I- $\lambda \rightarrow I$. The processes in I perform the action $\lambda$ evolving to I', in other words, I causes l' through $\lambda$. The intended dynamic meaning of such a rewriting rule is that the set I occurring in the current state can be replaced, after showing an event (labelled by) $\lambda$, by the set of processes $I$ '. We call these states distributed, since their components can be allocated in different places and can proceed on their own without requiring any centralized control, even when they synchronize. The description of parallel goal derivation relation is presented in Section 4.2 .

Even if the variables of a parallel goal may be shared by its sub-goals, substitutions generated by the proofs of the sub-goals are to be considered independent. This join AND-parallelism allows us to describe computations with decentralized loci of control in a simple manner by checking the compatibility of independently computed substitutions at join time only.

Since we are interested in giving semantics to goals which are communication-closed at the top level, the rewriting system has to be constrained so that an event goal can not be performed by a sequential process if the complementary event goal is not ready to be executed. This aim is reached by introducing a rule which filters only the well-formed transitions and it will be described in Section 4.3.

\subsection{Splitting a Delta Prolog parallel goal into a set of sequential processes}

\section{Definition 4.1. (Sequential Processes)}

We give the syntax of sequential processes:

$$
\text { SP: }:=\langle\square, \theta\rangle /\langle\mathrm{g}, \theta\rangle /\langle\text { failure }\rangle /\left\langle\mathrm{SPlid}, \mathrm{g}^{\prime}\right\rangle /\langle\text { idlSP, g' }\rangle
$$

where $\square$ is the empty goal, $g$ is a sequential goal, $\theta$ is a substitution, $g^{\prime}$ is a goal, failure is a special 
configuration and tags lid and idl record the context in which a sequential process is set. The set of sequential processes SP is ranged over by sp, and its subsets are named I, J (possibly indexed).

Intuitively speaking, a sequential process is a sequential goal together with a substitution and an access path defining its location within the syntactical structure of the parallel goal. If the access path is not empty, the second component of the pair is a goal representing a continuation (what its ancestor has to do next). See also Example 4.1 below.

Now we describe how to map any pair (goal, substitution> into a (finite) set of sequential processes.

Definition 4.2. (From goals to sets of sequential processes)

Function dec $: \Gamma_{\mathrm{G}} \rightarrow \mathrm{fin}\left(\mathrm{2}^{\mathrm{SP}}\right)$ is defined by structural induction on goals.

\section{Sequential Goal)}

$$
\begin{array}{lll}
\operatorname{dec}(\langle\square, \theta\rangle)=\{\langle\square, \theta\rangle\} & \operatorname{dec}(\langle\text { failure }\rangle)=\{\text { failure }\rangle \\
\text { g is a sequential goal } & \text { implies } & \operatorname{dec}(\langle\mathrm{g}, \theta\rangle)=\left\{\left\langle\mathrm{g}, \theta_{\rangle}\right\}\right.
\end{array}
$$

The following rules apply only to parallel goals.

Sequential Composition)

$$
\begin{array}{cll}
\left\langle\mathrm{sp}, \mathrm{g}^{\prime}\right\rangle \in \operatorname{dec}(\langle\mathrm{g}, \theta\rangle) & \text { implies } & \left\langle\mathrm{sp}, \mathrm{g}^{\prime} ; \mathrm{g}^{\prime \prime}\right\rangle \in \operatorname{dec}\left(\left\langle\mathrm{g} ; \mathrm{g}^{\prime \prime}, \theta\right\rangle\right) \\
\langle\mathrm{sp}, \square\rangle \in \operatorname{dec}(\langle\mathrm{g}, \theta\rangle) & \text { implies } & \left\langle\mathrm{sp}, \mathrm{g}^{\prime}\right\rangle \in \operatorname{dec}\left(\left\langle\mathrm{g} ; \mathrm{g}^{\prime}, \theta\right\rangle\right) \\
\text { lel Composition }) & & \\
\mathrm{sp} \in \operatorname{dec}(\langle\mathrm{g}, \theta\rangle) & \text { implies } & \langle\text { splid, } \square\rangle \in \operatorname{dec}\left(\left\langle\mathrm{g} \mid \mathrm{g}^{\prime}, \theta\right\rangle\right) \\
& \text { and } & \langle\text { idlsp, } \square\rangle \in \operatorname{dec}\left(\left\langle\mathrm{g}^{\prime} \| \mathrm{g}, \theta\right\rangle\right)
\end{array}
$$

Parallel Composition)

The configurations for the empty goal, failure and for a sequential goal are singleton sets of sequential processes. The Sequential Composition rule simply states that the decomposition recursively splits the left component only and that the rofle of the right component is to contribute to the continuation. Finally, the sequential processes derived from a parallel goal are exactly those derived by its two components, augmented by tags lid or idl, and with the empty goal as continuation, since nothing has to be done after the parallel goal has been solved.

\section{Example 4.1.}

Consider the parallel goal $\left(\left(\left(\mathrm{g}_{1} \| \mathrm{g}_{2}\right) ; \mathrm{g}_{3}\right) \| \mathrm{g}_{4}\right) ; \mathrm{g}_{5}$, where all the $\mathrm{g}_{\mathrm{i}}$ are sequential goals. Given a substitution $\theta$, we have:

i) $\operatorname{dec}\left(\left\langle\mathrm{g}_{\mathrm{i}}, \theta\right\rangle\right)=\left\{\left\langle\mathrm{g}_{\mathrm{i}}, \theta_{\rangle}\right\} \quad \mathrm{i}=1,2, \ldots, 5\right.$;

by Sequential Goal rule

ii) $\operatorname{dec}\left(\left\langle\mathrm{g}_{1} \| \mathrm{g}_{2}, \theta\right\rangle\right)=\left\{\left\langle\mathrm{g}_{1}, \theta\right\rangle\right.$ lid, $\left.\square\right\rangle,\left\langle\right.$ idk $\left.\left.\left.\mathrm{gg}_{2}, \theta\right\rangle, \square\right\rangle\right\}$

by i) and Parallel Composition rule

iii) dec $\left(\left\langle\left(g_{1} \| g_{2}\right) ; g_{3}, \theta\right\rangle\right)=\left\{\left\langle\left\langle g_{1}, \theta\right\rangle \mid i d, g_{3}\right\rangle,\left\langle\right.\right.$ idk $\left.\left.\left.g_{2}, \theta\right\rangle, g_{3}\right\rangle\right\}$

by ii) and Sequential Composition rule

iv) $\left.\operatorname{dec}\left(\left\langle\left(g_{1} \| g_{2}\right) ; g_{3}\right) \| g_{4}, \theta\right\rangle\right)=\left\{\left\langle\left\langle g_{1}, \theta\right\rangle \mid i d, g_{3}\right\rangle|i d, \square\rangle,\left\langle\right.\right.$ idl $\left.\left.\left.g_{2}, \theta\right\rangle, g_{3}\right\rangle|i d, \square\rangle,\left\langle i d \mid g_{4}, \theta\right\rangle, \square\right\rangle$

by i), iii) and Parallel Comp. rule

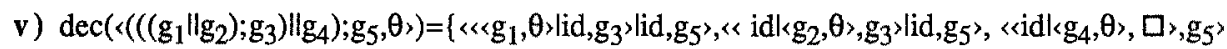

by iv) and Sequential Comp. rule

Note that function dec is injective, but it is not surjective since there are sets of sequential processes which are not obtainable via dec: for instance, $\left\{\left\langle\left\langle\mathrm{g}_{1}, \theta\right\rangle \mid \mathrm{id}, \square\right\rangle,\left\langle\mathrm{g}_{2}, \theta\right\rangle\right\}$.

Now we characterize the set of sequential processes corresponding to a possible configuration reachable from a starting goal. Let us consider the empty goal $\square$ and failure as legal Delta Prolog goals. We consider 
$\square$ as a legal goal because we use it for representing a terminated component process waiting for the termination of its brother, and the motivations for the introduction of failure as a legal goal are analogous.

Definition 4.3. (Complete sets of sequential processes)

Given a set $J$ of sequential processes, $J$ is complete iff there is a goal $g$ and a substitution $\theta$ such that $J$ is $\operatorname{dec}(\langle\mathrm{g}, \theta\rangle)$ up to substitutions.

The correspondence between $\operatorname{dec}(\langle\mathrm{g}, \theta\rangle)$ and $\mathrm{J}$ is up to substitutions because sequential processes generated by a parallel goal work in separate environments and thus their substitutions may be different.

\subsection{Rewriting rules for Parallel Goals}

Before giving the rewriting rules for parallel goals, we need an auxiliary definition. According to Delta Prolog specification, a parallel goal terminates if and only if all its sequential processes have succeeded and the computed substitutions are compatible.

Given a vector $\mathbf{X}$ of variables $\left\langle\mathrm{x}_{1}, \ldots, \mathrm{x}_{\mathbf{n}}\right\rangle$ and a substitution $\theta$, we say that $\mathbf{X} \theta=\left\langle\mathrm{x}_{1} \theta, \ldots, \mathrm{x}_{\mathbf{n}} \theta\right\rangle$ where $\forall \mathrm{x}_{\mathrm{i}} \notin \operatorname{Dom}(\theta): \mathrm{x}_{\mathrm{i}} \theta=\mathrm{x}_{\mathrm{i}}$

\section{Definition 4.4. (Substitution Unifier)}

Given two substitutions $\theta_{1}$ and $\theta_{2}$, let $X_{i}=\left\{v_{j} \mid v_{j} \notin \operatorname{Dom}\left(\theta_{i}\right) \wedge \exists v_{k} / t_{k} \in \theta_{i}: v_{j} \in \operatorname{Var}\left(t_{k}\right)\right\}$ with $i=1,2$. Let $X$ be the vector obtained from $\cup_{i=1,2} \operatorname{Dom}\left(\theta_{i}\right) \cup X_{i}$.

$\theta_{1}$ and $\theta_{2}$ are compatible if and only if $\exists \sigma=\operatorname{mgu}\left(X \theta_{1}, X \theta_{2}\right)$ and $\sigma$ is called the substitution unifier (SU) of $\theta_{1}$ and $\theta_{2}$.

Note that the definition above implies that: $\theta_{1} \sigma=\theta_{2} \sigma$, since the most general instance of $\left(\mathbf{X} \theta_{1}, \mathbf{X} \theta_{2}\right)$ is $\mathbf{X} \theta_{1} \sigma=\mathbf{X} \theta_{2} \sigma$.

\section{Example 4.2.}

Given $\theta_{1}=\{x / 2, y / f(s, z)\}$ and $\theta_{2}=\{x / w, y / f(t, 2)\}$, the sets $X_{1}$ and $X_{2}$ are $\{s, z)$ and $\{w, t\}$, respectively. Thus $X=\langle x, y, z, s, w, t\rangle, X \theta_{1}=\langle 2, f(s, z), z, s, w, t\rangle$ and $X \theta_{2}=\langle w, f(t, 2), z, s, w, t\rangle$. The more general instance of them is $\langle 2, f(t, 2), 2, t, 2, t\rangle$ and the unifying substitution $\sigma$, i.e. their $S U$ is $\sigma=$ $\{z / 2, s / t, w / 2\}$.

\section{Definition 4.5. (Labels)}

The set $\Lambda_{\mathrm{D}}$ of labels, ranged over by $\lambda_{\mathrm{D}}$, is the set $\{\theta\} \cup\{\mathrm{T}$ !e $\} \cup[\mathrm{T}$ ? $]$.

Notation: the application of a substitution $\sigma$ to a sequential process SP, denoted by SPo, is defined as follows:

$$
\begin{array}{lll}
\langle\square, \theta\rangle \sigma=\langle\square, \theta \sigma\rangle & \langle\mathrm{g}, \theta\rangle \sigma=\langle\mathrm{g} \sigma, \theta \sigma\rangle & \langle\text { failure }\rangle=\langle\text { failure }\rangle \\
\left\langle\text { SPlid, } g^{\prime}\right\rangle \sigma=\left\langle\text { SPolid, } g^{\prime}\right\rangle & \text { id }\left|S P, g^{\prime}\right\rangle \sigma=\left\langle\text { idlSP } \sigma, g^{\prime}\right\rangle &
\end{array}
$$

and the set $\{S P \sigma \mid S P \in I\}$ is denoted by $I \sigma$. We sometimes denote the set $\{<s p, g>\mid s p \in I\}$ by the pair $\langle I, g\rangle$. Besides, in the following definition, $g$ stands for a goal $g$ or the empty goal $\square$.

Definition 4.6. (Rewriting derivation relation for parallel goals)

The parallel goal derivation relation $\mathrm{I}_{1}-\lambda_{\mathrm{D}} \rightarrow \mathrm{I}_{2}$ is defined as the least relation satisfying the following 
axioms and inference rules.

Join)

$\theta_{1}$ and $\theta_{2}$ are compatible with $S U \sigma$

implies

$\left\{\mathrm{sp}_{1}, \mathrm{sp}_{2}\right\}-\sigma \rightarrow \operatorname{dec}\left(\left\langle(\mathrm{g}) \theta_{1} \sigma, \theta_{1} \sigma\right\rangle \quad\right.$ where $\mathrm{sp}_{1}=\left\langle\left\langle\square, \theta_{1}\right\rangle\right.$ lid, g $\mathrm{g}$ and $\left.\mathrm{sp}_{2}=\left\langle\mathrm{id} \mid<\square, \theta_{2}\right\rangle, \mathrm{g}\right\rangle$

$\mathrm{sp}_{1}=\langle\langle$ failure $\rangle$ lid, $\mathrm{g}\rangle$ and $\left.\mathrm{sp}_{2}=\left\langle\mathrm{id} \mid \nabla, \theta_{2}\right\rangle, \mathrm{g}\right\rangle \quad$ or

$\mathrm{sp}_{1}=\langle$ idk $k$ failure $\left.\rangle, \mathrm{g}\right\rangle$ and $\mathrm{sp}_{2}=\left\langle\left\langle\square, \theta_{2}\right\rangle \mathrm{lid}, \mathrm{g}\right\rangle \quad$ or

Act)

$\mathrm{sp}_{1}=\langle\langle$ failure $\rangle$ id, $g\rangle$ and $\mathrm{sp}_{2}=\langle$ id $|$ failure $\left.\rangle, g\right\rangle \quad$ implies $\quad\left\{\mathrm{sp}_{1}, \mathrm{sp}_{2}\right\}-\varepsilon \rightarrow\{$ ffailure $\left.\rangle\right\}$

$$
\begin{aligned}
\langle\mathrm{g}, \theta\rangle=\lambda_{\mathrm{G}} \Rightarrow & \left.\left\langle\mathrm{g}^{\prime}, \theta^{\prime}\right\rangle /\left\langle\square, \theta^{\prime}\right\rangle / \text { failure }\right\rangle \\
& \text { implies } \quad\{\langle\mathrm{g}, \theta\rangle\}-\lambda_{\mathrm{G}} \rightarrow \operatorname{dec}\left(\left\langle\mathrm{g}^{\prime}, \theta^{\prime}\right\rangle\right) /\left\{\left[\square, \theta^{\prime}\right\rangle\right\} /\{\langle\text { failure }\rangle\}
\end{aligned}
$$

Async)

$$
\begin{array}{lll}
I_{1}-\lambda_{D^{-}}>I_{2} & \text { implies } & I_{1} \text { lid, g }-\lambda_{D^{-}}>\left\langle I_{2} \text { lid, } g\right\rangle \\
& \text { and } & \text { idilI }, g\rangle-\lambda_{D} \rightarrow\left\langle\text { idlI }_{2}, g\right\rangle
\end{array}
$$

Sync)

$$
\begin{aligned}
& \begin{array}{lllll}
\mathrm{I}_{1}-\lambda_{1} \rightarrow \mathrm{I}_{1} & \text { and } & \mathrm{I}_{2}-\lambda_{2}-\mathrm{I}_{2} & \text { and } & \lambda_{1}=\mathrm{T}_{1} ! \mathrm{e} \text { and } \lambda_{2}=\mathrm{T}_{2} \text { ?e } \\
& \text { and } & \mathrm{mgu}\left(\mathrm{T}_{1}, \mathrm{~T}_{2}\right)=\sigma & \text { and } & \mathrm{T}_{\mathrm{i}} \sigma \text { is ground, } \mathrm{i}=1,2
\end{array} \\
& \text { implies } \quad\left\langle\mathrm{I}_{1} \text { lidu } \mathrm{idII}_{2}, g\right\rangle-\sigma \rightarrow\left\langle\left(\mathrm{I}_{1}^{\prime} \text { lid } \cup \text { idII' }{ }_{2}\right) \sigma, g\right\rangle \\
& \text { and }\left\langle\mathrm{I}_{2} \text { lidu idlI }{ }_{1}, g\right\rangle-\sigma \rightarrow\left\langle\left(\mathrm{I}_{2} \text { lid } \cup \text { idII' }{ }_{1}\right) \sigma, g\right\rangle
\end{aligned}
$$

\section{REMARKS}

Join) As soon as both components of a parallel goal terminate, the continuation of the parallel goal is enabled and its substitution updated. If $\theta_{1}$ and $\theta_{2}$ are not compatible, then the set $\left\{\mathrm{sp}_{1}, \mathrm{sp}_{2}\right\}$ represents a deadlocked state. If one of the components (or both) has failed, then the whole system fails. Note that the failure of a parallel goal is detected at join time only.

Act) This is essentially an import rule for sequential goals. Everything a sequential goal can perform in the transition system for sequential goals can be also performed by the corresponding singleton set in the rewriting system for parallel goals.

Async) From the premise that a set $J$ of sequential processes performs an action, we can infer that the same set can perform that action in any context.

Sync) The communication mechanism is handshake. Substitution $\sigma$ is applied to the set of sequential processes in order to keep trace of the bindings generated by the communication.

\subsection{Rewriting Rules for Systems}

Given a Delta Prolog program p, an initial goal g and the empty substitution $\varepsilon$, we describe the system $S_{\mathrm{p}, \mathrm{g}}$ with a suitable rewriting system. The rewriting rules for system $S_{\mathrm{p}, \mathrm{g}}$ are those of the previous rewriting system without the rules for performing asynchronous event goals. In other words, we introduce a closure condition stating that a goal must be "communication closed" at the top-level, i.e. unable to perform an event-communication with an external partner. Since we are considering programs and goals ready to be executed, we exclude possible open communications at all. 
Definition 4.7. (Rewriting derivation relation for systems)

The system derivation relation $I_{1}=\lambda_{D} \Rightarrow I_{2}$ is defined as the least relation satisfying the following inference rule:

\section{Communication-Closed)}

$$
\begin{array}{lll}
\mathrm{I}_{1}-\lambda_{\mathrm{D}} \rightarrow \mathrm{I}_{2} & \text { and } & \lambda_{\mathrm{D}} \neq \mathrm{T} \text { !e and } \lambda_{\mathrm{D}} \neq \mathrm{T} \text { ? } \\
& \text { implies } & \mathrm{I}_{1}=\lambda_{\mathrm{D}} \Rightarrow \mathrm{I}_{2}
\end{array}
$$

Note that this filtering rewriting rule is very close to the rule for the restriction operator of CCS [17]. Instead of introducing a new rewriting system for filtering such derivations, we could have added a syntactic construct ".l" and denote with g the communication closed goal corresponding to a goal g and we could have imposed that the initial goal were a restricted goal.

\section{Example 4.3.}

Let us consider the following program $\mathrm{p}$, where $\mathrm{T}$ is a ground term:

$$
\mathrm{A} \leftarrow(\mathrm{T} ! \mathrm{e} ; \mathrm{T} ? \mathrm{f}) ; \mathrm{A} \quad \oplus \quad(\mathrm{B} \leftarrow \mathrm{T} ! \mathrm{f} ; \mathrm{B} \oplus \mathrm{C} \leftarrow \mathrm{T} \text { ? } ; \mathrm{C})
$$

Let $A \|(B \| C)$ be the initial goal. The initial set of sequential processes is $\operatorname{dec}(<A \|(B \| C), \varepsilon)$. From the definition of the dec function we have that:

$$
\operatorname{dec}(\langle A \|(B \| C), \varepsilon\rangle)=\{\langle\langle A, \varepsilon\rangle \mid \operatorname{id}, \square\rangle,\langle\operatorname{idk}\langle\langle B, \varepsilon\rangle \mid i d, \square\rangle, \square\rangle,\langle\operatorname{id} \mid\langle i d \mid\langle C, \varepsilon\rangle, \square\rangle, \square\rangle\} .
$$

The first component of the set can make the following derivation:

$$
\{\langle A, \varepsilon\rangle|\mathrm{id}, \square\rangle\}=\varepsilon \Rightarrow\{\langle(\mathrm{T} ! \mathrm{e}: \mathrm{T} \text { ?f);A, } \varepsilon \gg \text { lid, } \square\rangle\}
$$

Below we give the complete deduction leading to that transition:
i) $\langle A: A \leftarrow(T ! e .: T ? f) ; A, \varepsilon\rangle-\varepsilon \rightarrow\rangle_{p}\langle(T ! e . \therefore T ? f) ; A, \varepsilon\rangle$
for Head Unification, since $\varepsilon=\operatorname{mgu}(\mathrm{A}, \mathrm{A})$
ii) $\langle A: p, \varepsilon\rangle-\varepsilon \rightarrow\rangle_{p}\langle(T ! e \therefore T ? f) ; A, \varepsilon\rangle$ by i) and Clause Selection
iii $\langle A, \varepsilon\rangle=\varepsilon \Rightarrow\langle(T$ !e $\therefore$ T?f $) ; A, \varepsilon\rangle$ by ii) and Goal Derivation
iv) $\{\langle A, \varepsilon\rangle\}-\varepsilon \rightarrow\{\langle(T ! e . \therefore T$ f $) ; A, \varepsilon\rangle\}$ by iii) and $A c t$
v) $\{\langle A, \varepsilon>$ lid, $\square\rangle\}-\varepsilon \rightarrow\{\langle(T$ te $: T$ ? $f) ; A, \varepsilon>$ lid, $\square\rangle\}$
by iv) and Async
vi $\{\langle\langle A, \varepsilon\rangle|$ id, $\square\rangle\}=\varepsilon \Rightarrow\{\langle(T ! e . . T ? f) ; A, \varepsilon\rangle \mid$ id, $\square\rangle\}$
by v) and Communication Closed

In an absolutely analogous manner, it is possible to derive the following:

$$
\begin{aligned}
& \{\text { idk }\langle B, \varepsilon>\text { lid, } \square\rangle, \square\rangle\}=\varepsilon \Rightarrow\{\text { idk }\langle T| f ; B, \varepsilon>\text { lid, } \square\rangle, \square\rangle\} \text { and } \\
& \text { \{ <idkidk } \mathrm{C}, \varepsilon\rangle, \square\rangle, \square\rangle\}=\varepsilon \Rightarrow\{\text { idkidk } T \text { ?e;C, } \varepsilon\rangle, \square\rangle, \square\rangle \text { \}. }
\end{aligned}
$$

The derivation corresponding to the communication between processes $\mathrm{A}$ and $\mathrm{B}$ follows:
a) (T?f, $\varepsilon\rangle=T$ ? $\Rightarrow\langle\square, \varepsilon\rangle$
for Event Goal
b) $\langle$ (Tle. $\therefore \mathrm{T}$ f $), \varepsilon\rangle, \square\rangle=\mathrm{T}$ ? $\Rightarrow\langle\square, \varepsilon\rangle$
by a) and Choice Goal
c) $\langle$ (T!e $\therefore \mathrm{T} ? \mathrm{f}) ; \mathrm{A}, \varepsilon\rangle=\mathrm{T}$ ? $\Rightarrow\langle\mathrm{A}, \varepsilon\rangle$
by b) and Sequential Composition
d) $\{\langle(T$ te $\therefore \mathrm{T} ? \mathrm{f}) ; \mathrm{A}, \varepsilon>\mathrm{id}, \square\rangle\}-\mathrm{T} ? \mathrm{f} \rightarrow\rangle\{\langle\mathrm{A}, \varepsilon>\mathrm{id}, \square\rangle\}$ by c) and $A c t$

Analogously, we derive:

e) $\{$ idd $\mid\langle T$ !f; $B, \varepsilon>\mid i d, \square\rangle, \square\rangle\}-T$ !f $\rightarrow>\{$ id $\mid\langle B, \varepsilon>|$ id, $\square\rangle, \square\rangle\}$.

Moreover, we have:

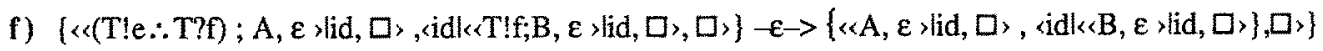
by d), e) and Sync

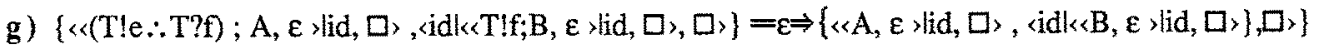
by ) and Communication Closed 
Quite similarly, it is possible to derive the following:

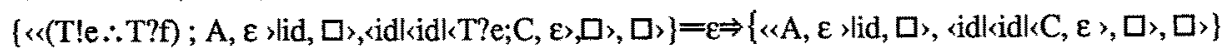

Our rewriting system is asynchronous. Actually, the derivations of a system are independent of those sequential processes which are concurrent with the rewritten ones, but inactive. In other words, each rule is context-independent.

Property 4.1. (Asynchrony of system derivation relation)

If $I_{1}=\lambda D \Rightarrow I_{2}$ is a derivation, there exists a set of sequential processes $J$ such that $J \cap I_{1}=\varnothing$ and $J \cup I_{1}$ is complete. Furthermore, for every such $\mathrm{J}$ we have also that $\mathrm{J} \cap \mathrm{I}_{2}=\varnothing$ and that $\mathrm{J} \cup \mathrm{I}_{2}$ is a complete set of sequential processes.

Proof. Immediate by induction on the structure of the proof of the derivation.

\section{A Delta Prolog System as a 1-Safe P/T NeT}

Now we build a 1-safe P/T net given a Delta Prolog program p, an initial goal g to be proved and the empty substitution $\varepsilon$. In the following, we use standard definitions of Petri net theory $[4,24]$. Roughly, the places of the net are the sequential processes belonging to $\operatorname{dec}(\langle\mathrm{g}, \varepsilon\rangle)$ and all those reachable from this starting set. The transitions are essentially the rewriting rules described in the previous section. The flow (causal) relation relates those sequential processes which are the left-hand side of a rewriting rule to the rewriting rule (transition) itself, and the rewriting rule to the new set of sequential processes composing the righthand side of the rule.

Definition 5.1. (From Delta Prolog systems to nets)

The set of places $\mathrm{S}$, the set of transitions $\mathrm{T}$, the flow relation $\mathrm{F}$ and the initial marking $M_{0}$ of the P/T net $\Sigma_{\mathrm{p}, \mathrm{g}}=\left\langle\mathrm{S}, \mathrm{T}, \mathrm{F}, M_{0}\right\rangle$ associated to system $S_{\mathrm{p}, \mathrm{g}}$ are defined as the least sets, relation and function satisfying the following inference rules:

- $\operatorname{sp} \in \operatorname{dec}(\langle\mathrm{g}, \varepsilon\rangle)$ $\mathrm{sp} \in \mathrm{S}$

- $\mathrm{I}_{1} \subseteq \mathrm{S} \quad$ and $\left(\mathrm{I}_{1}=\lambda_{\mathrm{D}} \Rightarrow \mathrm{I}_{2}\right) \in \mathrm{T}$

- $\left(I_{1}=\lambda_{D} \Rightarrow I_{2}\right) \in T$ implies

and $\mathrm{I}_{1}=\lambda_{\mathrm{D}} \Rightarrow \mathrm{I}_{2}$ and implies $\mathrm{I}_{1} \mathrm{~F}\left(\mathrm{I}_{1}=\lambda_{\mathrm{D}} \Rightarrow \mathrm{I}_{2}\right)$ and

$$
M_{0}(\mathrm{sp})=1
$$

implies

$\mathrm{I}_{2} \subseteq \mathrm{S}$ and $\forall \mathrm{sp} \in \mathrm{I}_{2} \backslash \operatorname{dec}\left(\langle\mathrm{g}, \varepsilon) M_{0}(\mathrm{sp})=0\right.$

Note that $\Sigma_{p, g}$ is indeed a P/T net since $\triangleleft, T, F$, is a net (it satisfies the condition $S \cap T=\varnothing$ ) and $M_{0}$ is a marking. The condition which says that $\forall t \in T \cdot t \neq \varnothing$ and $t^{*} \neq \varnothing$ is satisfied (third rule).

A transition $t \in T$ is enabled by a marking $M$ iff $\forall s \in t, M(s) \geq 1$. Furthermore, the occurrence of a transition $t$ produces the marking $M^{\prime}\left(M\left[\mathrm{t}>M^{\prime}\right)\right.$ :

$$
M^{\prime}(\mathrm{s})= \begin{cases}M(s) & \text { if } s \notin\left(\mathrm{t} \cup \mathrm{t}^{*}\right) \text { or } s \in\left(\mathrm{t} \cap \mathrm{t}^{*}\right) \\ M(\mathrm{~s})-1 & \text { if } \mathrm{s} \in \mathrm{t} \backslash \mathrm{t}^{*} \\ M(\mathrm{~s})+1 & \text { if } \mathrm{s} \in \mathrm{t} \backslash \mathrm{t}\end{cases}
$$


$\left[M_{0}>\right.$ denotes the set of all the possible markings $M$ reachable from $M_{0}$ by successive firing of enabled transitions. $M$ a 1 -safe marking iff $\forall \mathrm{s} \in \mathrm{S}, M(\mathrm{~s}) \leq 1$. The P/T net $\Sigma$ is $l$-safe iff $\forall M \in\left[M_{O^{2}}, M\right.$ is 1 -safe. Finally, we are going to prove that $\Sigma_{\mathrm{p}, \mathrm{g}}$ is 1 -safe.

\section{Theorem 5.1.}

Given a program p, a goal $\mathrm{g}$ and the substitution $\varepsilon$, the P/T net $\Sigma_{\mathrm{p}, \mathrm{g}}$ is 1-safe.

Proof. Since the P/T net $\Sigma_{\mathrm{p}, \mathrm{g}}$ is 1-safe if and only if $\forall M \in\left[M_{0}>M\right.$ is 1-safe, the proof is by induction on the set $\left[M_{0}\right\rangle$. At each step of induction we also prove that the set $P=\{\mathrm{s} \in \mathrm{S} \mid M(\mathrm{~s})=1\}$ is complete, in order to inherit the results of Property 4.1. By Definition 5.1, the initial marking $M_{0}$ is 1 -safe and the set $P_{0}=\left\{\mathrm{s} \in \mathrm{S} \mid M_{0}(\mathrm{~s})=1\right\}=\operatorname{dec}(\mathrm{g}, \varepsilon)$ is complete. Let us suppose, by inductive hypothesis, that a reachable marking $M$ is 1-safe and that its corresponding set $P$ is complete. Let $\mathrm{t}=\mathrm{I}_{1}=\lambda_{\mathrm{D}} \Rightarrow \mathrm{I}_{2}$ a $M$ enabled transition and $M\left[\mathrm{t}>M^{\prime}\right.$. We have to prove that the produced marking $M^{\prime}$ is 1 -safe and that the corresponding set $P^{\prime}$ is complete. Let $\mathrm{J}=P \mathrm{~V}_{1}$. By definition of produced marking, $M^{\prime}$ on the set of places $\mathrm{I}_{1} \mathrm{I}_{2}$ holds 0 , on the set of places $\mathrm{I}_{2} \cup \cup \mathrm{I}_{1} \cap \mathrm{I}_{2}$ holds 1 , while for the set of places $\mathrm{J} \cap \mathrm{I}_{2}$ marking $M^{\prime}$ would hold 2. But, by Property 4.1 , we have that $\mathrm{J}_{2} \mathrm{I}_{2}=\varnothing$ - thus marking $M^{\prime}$ is 1 -safe - and finally that $P^{\prime}=$ $\mathrm{JU}_{2}$ is complete.

We eventually define the operational semantics of a Delta Prolog system $S_{\mathrm{p}, \mathrm{g}}$. The operational semantics can not be defined simply as the set of all the computed answer substitutions since occurrence sequences may be infinite or, though terminal (i.e. finite with no transition enabled by the last marking), may lead to deadlock. The possible last markings of a terminal occurrence sequence (o.s. for short) may be:

- the single place $\{\square, \theta\rangle\}$, which corresponds to a correct answer substitution $\theta$,

- the single place ( failure)\}, i.e. a failure occurs since an atom goal can not unify with any clause,

- a set I of places, which stands for a deadlock possibly due to (more than) one of the following reasons:

- the non-existence of a compatible substitution (in case of termination of a parallel goal),

- the inability to unify terms in a matching pair of event goals,

- the possible partner for communication is terminated or failed.

Summing up, we can say that the operational semantics of a Delta Prolog system $S_{\mathrm{p}, \mathrm{g}}$ is:

$$
\begin{aligned}
& {\left[S_{\mathrm{p}, \mathrm{g}} \mathbb{J}_{\mathrm{o}}=\left\{\theta \mid \xi \text { is a terminal o.s. of } \Sigma_{\mathrm{p}, \mathrm{g}} \text { and last }(\xi)=\left(\square, \theta_{\rangle}\right]\right\}\right.} \\
& \left.\left.\cup \quad \text { (fail } \mid \xi \text { is a terminal o.s. of } \Sigma_{p, g} \text { and last }(\xi)=\text { \{failure }\right]\right\} \\
& \cup \quad\left\{\Delta \mid \xi \text { is a terminal o.s. of } \Sigma_{p, g} \text { and }|\operatorname{last}(\xi)| \geq 2\right\} \\
& \cup \quad\left\{\infty \mid \xi \text { is an infinite o.s. of } \Sigma_{p, g}\right\} \text {. }
\end{aligned}
$$

\section{FAIRNESS AND DEADLOCK}

We could be dissatisfied for this final state semantics since it is not adequate w.r.t. infinite computations (perpetual processes) in the sense that relevant features of behaviours are not dealt with. A better description of system $S_{\mathrm{p}, \mathrm{g}}$ should take into account all the actions performed (thus we know what the system is doing) and their causal relations, i.e. every computation is observed as the partial ordering of events it generates. Moreover, important properties, such as safety and liveness properties, can be observed by considering the overall evolution of the system.

Let us consider the following Delta Prolog program taken from [21]. A counter object is programmed 
allowing commands for incrementing and checking the value of a counter:

$$
\begin{array}{ll}
\text { counter }(\mathrm{S}) \leftarrow & \text { up(N)?mail; } \mathrm{U} \text { is } \mathrm{S}+\mathrm{N} \text {; counter(U) } \\
& \therefore \text { equal_to(S)?mail; counter(S). } \\
\text { producer } \leftarrow \quad \text { prod(X); } X \text { !mail; producer. }
\end{array}
$$

where prod is a predicate generating commands for the counter. An initial goal may be:

$$
\leftarrow \text { counter(0) II producer II producer. }
$$

It is trivial to observe that the computations of this Delta Prolog system may not terminate while still having a precise meaning: an infinite sequence of communications and updatings of the counter value. The denotation of programs which do not compute answer substitutions is represented by the set of all the occurrence sequences (sequential behaviour) or by the set of processes $[4,24]$ (concurrent behaviour) ${ }^{2}$. With these kinds of denotation, we are able to observe properties of perpetual processes like fairness and partial deadlock. The competition between two producers may be solved by the counter serving only one of them from a certain time onwards: this is a typical example of unfair computation. A situation of partial deadlock may arise if a producer sends an equal_to command to the counter process with an argument minor than the counter value: in this case the producer which has sent the message is deadlocked while the other producer and the counter may proceed.

A first attempt to express such properties in this framework may be the following. We look at an o.s. simply as the sequence of the sets of places with one token. Given a reachable marking $M$, let $P=$ $\{\mathrm{s} \in \mathrm{S} \mid M(\mathrm{~s})=1\}$; an o.s. $\xi=M_{0} \mathrm{t}_{1} M_{1} \mathrm{t}_{2} M_{2} \mathrm{t}_{3} M_{3} \ldots$ are observed as the sequence $P_{0} P_{1} P_{2} P_{3} \ldots$

Among the various notion of faimess (see ${ }^{[13]}$ for a detailed description of this issue), we just consider a couple of them. The first is called global fairness in [11] and communication fairness in [13]. It states that a transition $t=I_{1}=\lambda D \Rightarrow I_{2}$ which is always enabled will eventually be fired. This fact is expressed by the following formula:

$$
\operatorname{not}\left(\exists \mathrm{i} \in \mathrm{N}, \exists \mathrm{t} \in \mathrm{T}:{ }^{\mathrm{t}} \subseteq \cap_{\mathrm{j} \geq \mathrm{i}} P_{j}\right) \text {. }
$$

A second, more demanding notion of faimess (local faimess ${ }^{[11]}$ or process fairness ${ }^{[13]}$ ) concerns the fact that a place which can always (infinitely often, respectively) be involved in a firing, possibly within different transitions, will eventually be consumed. We represent this fact as:

$$
\begin{gathered}
\operatorname{not}\left(\exists \mathrm{i} \in \mathrm{N}, \exists \mathrm{s} \in \mathrm{S}: \mathrm{s} \in \cap_{\mathrm{j} \geq \mathrm{i}} P_{j} \text { and } \forall \mathrm{j} \geq \mathrm{i} \exists \mathrm{t} \in \mathrm{T},{ }^{*} \mathrm{t} \subseteq P_{j} \text { and } \mathrm{s} \in{ }^{*} \mathrm{t}\right) \\
\operatorname{not}\left(\exists \mathrm{i} \in \mathrm{N}, \exists \mathrm{s} \in \mathrm{S}: \mathrm{s} \in \cap_{\mathrm{j} \geq \mathrm{i}} P_{j} \text { and } \exists \text { infinitely many } \mathrm{j}, \mathrm{j} \geq \mathrm{i}, \exists \mathrm{t} \in \mathrm{T},{ }^{*} \mathrm{t} \subseteq P_{j} \text { and } \mathrm{s} \in{ }^{*} \mathrm{t}\right) \text {. }
\end{gathered}
$$

We can also write formulae stating that an o.s. is free of some kind of deadlock. For instance, a sequence $P_{0} P_{1} P_{2} P_{3} \ldots$, satisfying the following formula:

$$
\exists i \in N, \exists s \in S: s \in \cap_{j \geq i} P_{j} \text { and } g \circ a l(s)=g_{e} / g_{e} ; g / g_{c} \therefore g_{c}
$$

shows a deadlock due to a missing matching event goal, where goal: $\mathrm{S} \rightarrow \mathrm{G}$ is the function that extracts from a place (sequential process) the sequential goal it represents. Note that a sequential process of this kind could have partners that may never perform the complementary event goal. Thus a sequence with a partial deadlock may be locally fair, as well.

Another manner to define the operational semantics of a DP system $S_{\mathrm{p}, \mathrm{g}}$ consists in considering its processes, i.e. the pairs $\pi=\langle N, p\rangle$, where $N$ is an occurrence net and $p$ : BuE $\rightarrow$ SUT a labelling of $N$. For finite processes, we have that $\operatorname{Max}(N)$ may be $\{[\square, \theta\rangle\}$, or $\{$ failure $\}$, or even a non-singleton set of

2 In order to better specify a system, it is necessary to consider structures which are less simplistic than sets, like event structures, synchronization trees or nondeterministic measurement systems. We will not be concerned with this aspect of the problem. 
places. For infinite processes, $\operatorname{Max}(\mathrm{N})$ denotes the set of places that do not fire from a certain point onwards. If a transition $t$ is such that $t \subseteq \operatorname{Max}(\mathrm{N})$, then $\langle\mathrm{N}, \rho$, is not globally fair. On the contrary, nothing we can say about local faimess. Even if a place $s$ belongs to $\operatorname{Max}(\mathrm{N})$, we do not know if there has always (infinitely often) been some transition $t$, which may be time-dependent, such that $s \in$ " $t$.

This is a first possible approach to the problem of describing properties of perpetual processes. Further research would be mandatory to better investigate this subject.

\section{CONCLUSIONS}

In this paper a distributed, net oriented semantics for a Concurrent Logic Language is presented for the first time. We have studied a model of computation based on AND-parallelism together with an explicit mechanism of synchronization. Delta Prolog has been chosen as a case study.

In order to give a truly distributed semantics, we resorted to techniques developed for imperative and functional languages $[8,10,11,19]$ and adapted them to the case of logic languages. This approach allows us to represent causal relations among processes in a very direct way and to address the problem of observing properties that are intrinsically based on causality.

\section{Acknowledgments}

We would like to thank Pierpaolo Degano for many valuable discussions and suggestions. Thanks also to Catuscia Palamidessi, Ugo Montanari and Franco Turini for their comments and encouragement.

\section{REFERENCES}

[1] J.N. Aparicio, J.C. Cunha, L.F. Monteiro, L.M. Pereira, "The Specification of Delta Prolog", Draft manuscript, June 1987.

[2] K.R. Apt, M.H. van Emden, "Contribution to the Theory of Logic Programming", J. ACM 29,3, 1982, pp. 841-862.

[3] J.W. de Bakker, J.N. Kok, "Uniform Abstraction, Atomicity and Contractions in the Comparative Semantics of Concurrent Prolog", Proc. of Int. Conf. on Fifth Generation Computer Systems, Tokyo, 1988, pp 347-355, Vol. II.

[4] E. Best, R. Devillers, "Sequential and Concurrent Behaviour in Petri Net Theory", Theoretical Computer Science 55, 1987, pp. 87-136.

[5] S.D. Brookes, C.A.R. Hoare, A.D. Roscoe, "A Theory of Communicating Sequential Processes", J. ACM 31, 3, 1984, pp.560-599.

[6] K.L. Clark, S. Gregory, "PARLOG: A Parallel Logic Programming Language", ACM Trans. on Prog. Lang. and Syst. 8, 1, Jan. 1986, pp. 1-49.

[7] J. Conery, D. Kibler, "Parallel Interpretations of Logic Programs", in Proc. ACM Conf. on Functional Prog. Languages and Comp. Architectures, 1981, pp. 163-170.

[8] P. Degano, R. De Nicola, U. Montanari, "A Distributed Operational Semantics for CCS based on Condition/Event Systems", Nota Interna B4-21, 1987. To appear in Acta Informatica.

[9] P. Degano, S. Diomedi, "A first order semantics of a connective suitable to express concurrency", in Proc. 2nd Logic Programming Workshop, Albufeira (Portugal), 1983, pp. 506-517.

[10] P. Degano, R. Gorrieri, S. Marchetti, "An Exercise in Concurrency: A CSP Process as a Condition/ Event System", in Proc. $8^{\text {th }}$ European Workshop on Applications and Theory of Petri Nets, 
Zaragoza, 1987, pp 31-50. To appear in Advances in Petri Nets 1988 (G. Rozenberg, ed.) LNCS, Springer 1988.

[11] P. Degano, U. Montanari, "Concurrent Histories: A Basis for Observing Distributed Systems", J.CSS 34, 1987, pp. 442-461.

[12] M. Falaschi, G. Levi, C. Palamidessi "A Synchronization Logic: Axiomatics and Formal Semantics of Generalized Horn Clauses", Information and Control, 60, 1-3 , pp.36-69.

[13] N. Francez, "Fairness", Text and Monographies in Computer Science (D. Gries, ed.), Springer, 1986.

[14] R. Gerth, M. Codish, Y. Lichtenstein, E.Y. Shapiro, "Full Abstract Denotational Semantics for Flat Concurrent Prolog", Proc. Logic in Computer Science, 1988, pp 320-333.

[15] C.A.R. Hoare, "Communicating Sequential Processes", C. ACM 21, 8, 1978, pp. 666-677.

[16] J.W. Lloyd, Foundations of Logic Programming, Springer 1984 (Second Edition 1987).

[17] R. Milner, "Notes on a Calculus for Communicating Systems", in Control Flow and Data Flow: Concepts of Distributed Programming (M. Broy,ed.), NATO ASI Series F, Vol. 14, Springer, 1984, pp. 205-228.

[18] L. Monteiro, "A proposal for distributed programming in logic", Implementations of Prolog - Ellis Horwood, 1984.

[19] E.-R. Olderog, "Operational Petri Net Semantics for CCSP", in Advances in Petri Nets 1987, (G. Rozenberg, ed.), LNCS 266, Springer 1987, pp. 196-223.

[20] L.M. Pereira, R. Nasr, "Delta Prolog: a distributed logic programming language", Proc. of FGCS, Tokyo, November 1984.

[21] L.M. Pereira, L.F. Monteiro, J. Cunha, J. Aparicio, M.C. Ferreira, Delta Prolog User's Manual Version 1.0, June 1987.

[22] L.M. Pereira, L. Monteiro, J.C.. Cunha, J.N. Aparicio, "Delta Prolog: a distributed backtracking extension with events", Proc. of 3rd International Conference on Logic Programming, July 1986.

[23] G.D. Plotkin, "A Structural Approach to Operational Semantics", Technical Report DAIMI FN-19, CS Department, University of Aarhus, 1981.

[24] W. Reisig, Petri Nets: An Introduction, EATCS Monograph in Computer Science, Springer, 1985.

[25] V.A. Saraswat, "The Concurrent Language CP: Definition and Operational Semantics", Proc. of the $14^{\text {th }}$ Symposium on Principles of Programming Languages, ACM, January 1987, pp. 49-62.

[26] E.Y. Shapiro, "Concurrent Prolog: a progress report", in Fundamentals of Artificial Intelligence (W. Bibel, Ph. Jorrand, eds.), LNCS 232, Springer, 1987, pp. 277-313.

[27] K. Ueda, Guarded Horn Clauses, Ph.D. Thesis, University of Tokyo, 1986, (also as Technical Report TR-103, ICOT 1986).

[28] M. van Emden, R.A. Kowalski, "The Semantics of Predicate Logic as a Programming Language", J. ACM, 23, 1976, pp. 733-742. 